\title{
Miglustat: A glycotransferase inhibitor for Covid-19 treatment
}

\section{Dean Tatlow}

Funding: The author(s) received no specific funding for this work.

Potential competing interests: The author(s) declared that no potential competing interests exist.

\begin{abstract}
Severe acute respiratory syndrome coronavirus 2 (SARS-CoV-2) undergoes blood type specific glycosylation which has implications for infection susceptibility and replication without detection from the immune system. SARS-CoV-2 hijacks the host cell glycotransferase resulting in spike protein glycosylation resembling blood type antigens. Infection risk correlates to blood types that do not have anti-A and/or anti-B antibodies similar to that seen for ABO blood type recipients. The universal recipient $A B$ is highly susceptible to infection lacking both anti-A and $B$ antibodies, whereas blood type $\mathrm{O}$ has both antibodies resulting in less risk of infection. Once infected, SARS-CoV-2 obtains the blood type specific glycosylation of the host resulting in an effective camouflage against immune system recognition. Decoding the link between blood type and coronavirus disease 2019 (COVID-19) susceptibility exposes a role for miglustat a glycosyltransferase inhibitor in treatment. Use of the FDA-approved glycosyltransferase inhibitor miglustat can inhibit spike protein glycosylation revealing the SARS-CoV-2 virus for immune system recognition.
\end{abstract}

Title

Miglustat: A glycotransferase inhibitor for Covid-19 treatment.

Dean Tatlow, Corinne Tatlow, Scarlet Tatlow and Savanah Tatlow

\section{INTRODUCTION}

The severe acute respiratory syndrome coronavirus 2 (SARS-CoV-2) spike protein is the main protein used in many vaccines, revealing its importance in immune system recognition of the SARS-CoV-2 virus. A recent paper by Grant et. al. showed the SARS-CoV-2 spike protein surface is highly shielded by glycans preventing antibody recognition. ${ }^{1}$ Since the virus hijacks the host cellular machinery, spike protein glycosylation would obtain a host blood type glycan surface. As mentioned by Grant et al the viral glycan shield may be composed of familiar host glycans. ${ }^{1}$ Another study dealing with SARS-CoV-1 has indicated glycotransferase activity resulting in A antigen variant of the ABO blood group with anti-A antibodies able to cause virus neutralization. ${ }^{2}$ Infection susceptibility may therefore be related to the ABO blood type recipients, and once infected the SARS-CoV-2 would obtain the host cell glycosylated coat becoming blood type specific.

The ABO blood-type is determined by the type of glycosylation found on the surface of red blood cells. The 
enzymes responsible for blood type glycosylation are known as glycotransferases. ${ }^{2}$ There are four possible blood types A, $\mathrm{B}, \mathrm{O}$ and $\mathrm{AB}$ while the $\mathrm{Rh}$ system is either Rh positive or negative. ${ }^{3}$ Blood-typing identifies individuals who are recipients and/or donors of red blood cells. Blood type recipients lack antibodies to donor red blood cells with AB+known as universal recipients. ${ }^{3}$ While donors lack either $\mathrm{A}$ and or B surface antigens glycosylation making O- individuals known as universal donors. ${ }^{3}$ The relationship to blood type and covid- 19 has been demonstrated by many papers with blood type $O$ found to have a decreased risk of morbidity and mortality associated with coronavirus disease 2019 (Covid-19) 4,5,6. The impact of glycosylation on the ability of antibodies to bind the SARS-CoV-2 spike protein plays a role not only in infection susceptibility but also in replication without detection by the immune system.

In this paper a new antiviral mechanism of action is proposed for miglustat that is different from inhibition of receptor binding. ${ }^{7,8} \mathrm{~A}$ main feature of SARS-Cov-2 is the avoidance of immune recognition by the protective glycan coat. ${ }^{1}$ The mechanism of action purpose in this paper of miglustat is treatment of SAR-CoV-2 by the removal of the protective glycan coat exposing the virus to immune system recognition (Figure 1). Miglustat is a FDA approved drug for the treatment of Gaucher disease and Niemann-Pick disease type $\mathrm{C}$ because of its alpha-glucosidase inhibition. ${ }^{9}$ Miglustat was shown to decrease the intracellular accumulation of glycosylcereride the glycolipid that accumulates in Gaucher disease. Sideeffects with miglustat are common and included diarrhea, weight loss, gastrointestinal upset, nausea and vomiting, anorexia, constipation, headache, tremor, dizziness, paresthesia, peripheral neuropathy, ataxia, visual problems, and memory loss. ${ }^{9}$ Side-effects are manageable since treatment duration with miglustat may be between 3-10days to correlate with the viral replication window.

\section{DISCUSSION}

The relationship of blood type susceptibility to SARS-CoV-2 infection is a result of host cell blood type glycosylation of the virus. ${ }^{4,5,6}$ The SARS-CoV-2 spike protein obtains a similar glycan coat as red blood cells, and infection susceptibility becomes related to blood type recipients. An AB blood type is universally susceptible to SARS-CoV-2 similar to the universal recipient status of $A B$ whereas blood type $O$ has less risk of infection. Reports have shown blood groups $A$ or $A B$ are at increased risk from SARS-CoV-2 infection versus those of blood group $O$ an $B{ }^{4,5,6}$ These results correlated with those seen in table 1 where $A B$ and $A$ have $100 \%$ to $88 \%$ susceptibility respectively to SARS-CoV-2, whereas bloodtype $B$ and $O$ are less susceptible with $55 \%$ and $46 \%$, respectively. The anti-A and anti-B antibodies of blood type $O$ individuals provide a barrier to infection from A, B, and AB blood type individuals infected with SARS-CoV-2. However, blood type O individuals can become infected by SARS-CoV-2 infected blood type O individuals. Once an individual is infected by SARS-CoV-2 they produce viruses with glycans matching the host blood type. The spike protein glycosylation of SARS-CoV-2, provides the virus with an effective camouflage against host immune system recognition. ${ }^{1} \mathrm{~A}$ blood type $\mathrm{O}$ individual can thus infect a blood type A individual, with the resultant virus replication producing blood type A SARS-CoV2. Blood type association with SARS-Cov-2 infection is underlined by virus hijacking host cell machinery enzymes involved in blood type glycosylation by glycotransferases indicating inhibition of these enzymes as a possible treatment for covid-19.

The evaluation of miglustat Covid-19 treatment effectiveness was previously investigated In Vitro revealing no impact in the diseaes. ${ }^{8}$ In a paper by Nunes-Santos et al, miglustat treatment had no impact on receptor binding of SARS- 
CoV-2 spike protein to the ACE2 receptor. ${ }^{8}$ In addition, although cytokine production was enhanced in both the miglustat treated spike protein and non-treated spike protein stimulation of peripheral blood mononuclear cells (PBMCs) there was no difference in cytokine production between them. ${ }^{8}$ Understanding the relationship between blood type and SARS-Cov-2 infection susceptibility and replication without immune system recognition reveals a mechanism explaining asymptomatic and presymptomatic patients with coronavirus. In these patients no immune response has been initiated suggesting a lack of cytokine production. A study by Long et al., confirms asymptomatic Covid-19 patients have no difference in cytokine production when compared to healthy individuals. ${ }^{9}$ The In Vitro model of spike protein stimulation of PBMCs by NunesSantos et al does not reflect In Vivo coronavirus infection, since In Vivo SARS-CoV-2 spike protein glycosylation prevents immune system recognition. ${ }^{1}$ Therefore, experiments revealing no difference in cytokine production with and without miglustat treatment are comparing two antigenic proteins. A proposed mechanism of action for miglustat in Covid-19 treatment is the inhibition of spike protein glycosylation resulting in SARS-CoV-2 recognition by the immune system. Evaluation of miglustat treatment for Covid-19 patients should illicit production of cytokines resulting in fever which will work as an early marker of treatment effectiveness. Finally, Covid-19 infected patients with Gaucher disease were initially suspected to be highly vulnerable to viral infection, however, reports have shown no hospitalization amongst this group. ${ }^{10}$ ${ }^{11}$ Whether glycotransferase inhibitors are playing a role in decrease hospitalization of gaucher disease patients has yet to be determined. ${ }^{10,11}$ Miglustat is a FDA approved medication for treatment of Gaucher disease at $100 \mathrm{mg}$ three times a day and Niemann-Pick Type $\mathrm{C}$ disease at $200 \mathrm{mg}$ three times day. ${ }^{12}$

The implication of extensive SARS-CoV-2 glycosylation is seen in the increased severity of Covid-19 in diabetic patients with high hemoglobin A1C. ${ }^{13} \mathrm{~A}$ study by Merzon et al showed pre-infected patients with a hemoglobin A1C of greater than $9 \%$ was a risk factor for Covid-19 severity. ${ }^{13}$ As with diabetic individuals where high hemoglobin A1C results in more extensive glycosylation of red blood cells, SARS-CoV-2 spike protein may be further glycosylated in this environment. Increased SARS-CoV-2 spike protein glycosylation would lead to an improved virus glycan shield, providing an effective barrier against immune system recognition.

\section{ACKNOWLEDGEMENTS}

Thank-you Stanley Vindevoghel

\section{REFERENCES}

1.Grant, O.C., Montgomery, D., Ito, K. et al. Analysis of the SARS-CoV-2 spike protein glycan shield reveals implications for immune recognition. Sci Rep 2020;10, 14991

2.Guillon $\mathrm{P}$, Clément M, Sébille V, et al.Inhibition of the interaction between the SARS-CoV spike protein and its cellular receptor by anti-histo-blood group antibodies.Glycobiology. 2008;18(12):1085-1093. 
3. Canadian blood services. Understanding your blood type. 2020. https://www.blood.ca/en/blood/donating-blood/whatsmy-blood-type

4. Hoiland, R. L., Fergusson, N. A., Mitra, A. R., Griesdale, D. E. G., Devine, D. V., Stukas, S., Cooper, J., Thiara, S., Foster, D., Chen, L. Y. C., Lee, A. Y. Y., Conway, E. M., Wellington, C. L., and Sekhon, M. S. The association of ABO blood group with indices of disease severity and multiorgan dysfunction in COVID-19. 2020;Blood Adv. 4, 4981- 4989.

5.Ellinghaus D, Degenhardt F, Bujanda L, et al; Severe Covid-19 GWAS Group. Genomewide association study of severe Covid-19 with respiratory failure.N Engl J Med. 2020.

6.M. B. Barnkob, A. Pottegård, H. Støvring, et al; Reduced prevalence of SARS-CoV-2 infection in ABO blood group O, Blood Advances 2020;4,4990-4993 .

7.Ahmad Abu Turab Naqvi, Kisa Fatima, Taj Mohammad, Urooj Fatima, Indrakant K. Singh, Archana Singh, Shaikh Muhammad Atif, Gururao Hariprasad, Gulam Mustafa Hasan, Md. Imtaiyaz Hassan, Insights into SARS-CoV-2 genome, structure, evolution, pathogenesis and therapies: Structural genomics approach, Biochimica et Biophysica Acta (BBA) Molecular Basis of Disease, 2020 Volume 1866, Issue 10.

8. Nunes-Santos CJ, Kuehn HS, Rosenzweig SD. N-Glycan Modification in Covid-19 Pathophysiology: In vitro Structural Changes with Limited Functional Effects. J Clin Immunol. 2020 Nov 27:1-10.

9. Long, Q. X., Tang X.J., Shi Q.L., et al. Clinical and immunological assessment of asymptomatic SARS-CoV-2 infections. Nat. Med. 2020; 26: 1200-1204.

10.Fierro L, Nesheiwat N, Naik H, et al. Gaucher disease and SARS-CoV-2 infection: Experience from 181 patients in New York.Mol. Genet. Metab. 2021;132:44-48.

11.A. Zimran, J. Szer, S. Revel-Vilk. Impact of Gaucher disease on COVID-19. Intern. Med. J. 2020; 50(7). 894-895.

12. Miglustat(Systemic). In: Lexi-Drugs. Lexi-Comp, Inc. Updated November 24, 2020. Accessed December 20, 2020.

13.Merzon E, Green I, Shpigelman M, et al. Haemoglobin A1C is a predictor of COVID-19 severity in patients with diabetes. Diabetes Metab Res Rev 2020. 


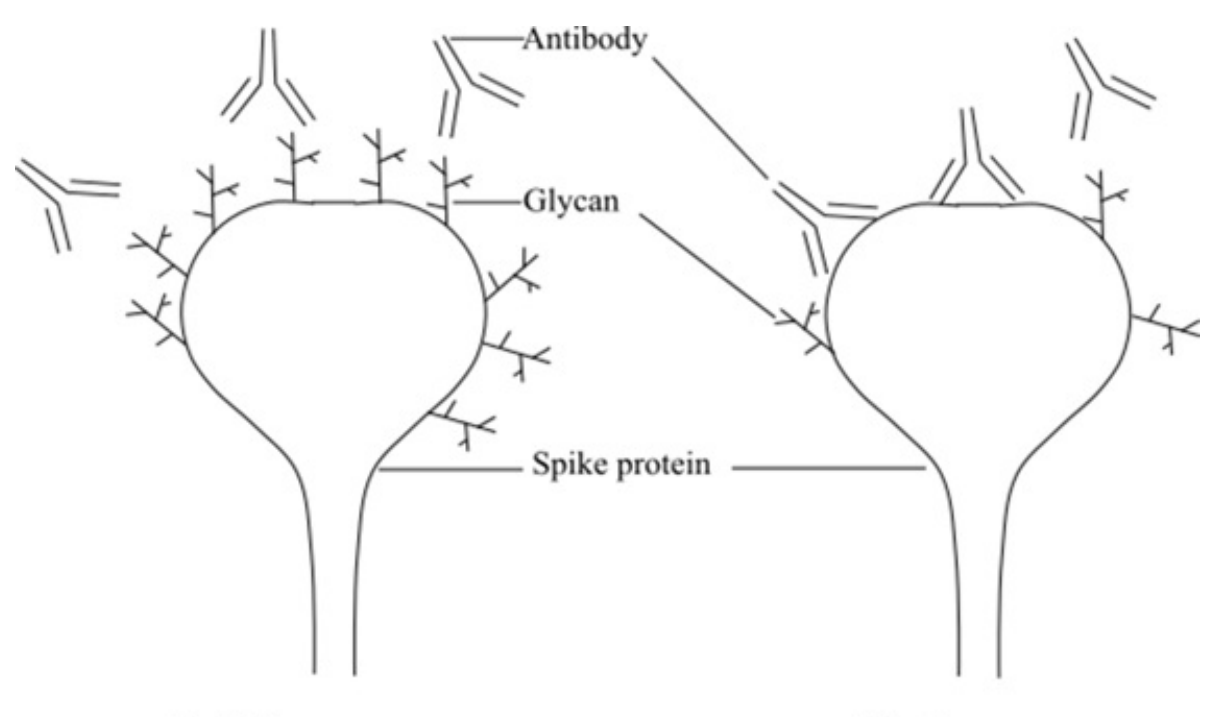

No Miglustat treatment

Migulstat treatment

Figure 1. Miglustat treatment of SARS-CoV-2 spike protein and immune system recognition.

\section{Donor Blood Types}

\begin{tabular}{|c|c|c|c|c|c|}
\hline 2 & $\mathrm{O}$ & B & A & $\mathrm{AB}$ & TOTAL \\
\hline 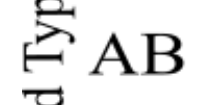 & $46 \%$ & $9 \%$ & $42 \%$ & $3 \%$ & $100 \%$ \\
\hline A & $46 \%$ & & $42 \%$ & & $88 \%$ \\
\hline D $B$ & $46 \%$ & $9 \%$ & & & $55 \%$ \\
\hline $\mathrm{O}$ & $46 \%$ & & & & $46 \%$ \\
\hline
\end{tabular}


Blood type percentage is representative of the Canadian population.

Table 1. Susceptibility to SAR-Cov-2 based on recipient blood types and their percentage represented in the population. 